stream to the transmitter. Fig. 2 shows the type of tracing obtained with a complete block of the deep veins of the left leg.
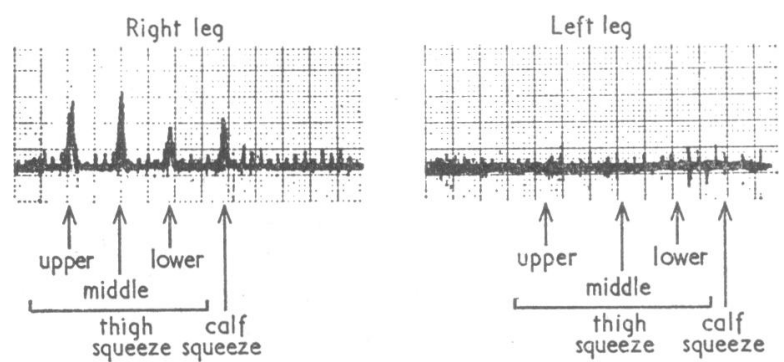

FIG. 2.- The ultrasound flowmeter record of a man with a total left deep vein block. Squeezing the calf or thigh on the right side increases the flow and produces an audible noise recorded side in upsuing (A wave) on the record Squeezing the left the left leg produced no change in flow indicating a blocked vein. (Reproduced by kind permission of the authors, D. S. Evans a

This method is quick and extremely simple and studies the important source of major emboli-the femoral and iliac veins and the vena cava. On the other hand, a disadvantage of the method is that flow in large collaterals or in a very large superficial vein will produce a sound similar to that produced by flow in a patent femoral vein. These circumstances may produce a false-negative reading. Early thrombi in the femoral vein sometimes propagate along the vein without producing any significant obstruction, and this type of thrombus may also be missed. Small thrombi in the calf veins, the tributaries of the profunda vein, or the internal iliac veins cannot be detected because they do not cause major vein obstruction. Despite these drawbacks, however, ultrasound is a valuable and rapid method of examining the major veins of a leg for large occlusive thrombi.

Perhaps some caution should be exercised while squeezing the leg to produce the increase in flow, as young thrombi are known to be very loose and friable; theoretically a thrombus might be broken free if the leg is squeezed too vigorously.

When the absence of flow suggests an occlusion of a major vein a phlebogram must still be performed because possibly the occlusion is old and not due to fresh, loose thrombosis. Before proceeding to any form of treatment more detailed information is needed concerning the nature and extent of the thrombus.

\section{Selection of Tests}

Phlebography is undoubtedly the most accurate technique ; it is essential before any surgical treatment and is important and helpful before deciding on medical treatment, such as thrombolysis.

${ }^{125}$ I-fibrinogen is a very accurate technique for detecting thrombus below the upper thigh, but there is a delay of 6-24 hours after giving the fibrinogen before the count rates are of diagnostic value. However, the results from using this method may be available earlier if phlebography cannot be carried out immediately, and the whole technique can be carried out by a technician. I use the method extensively for confirming the diagnosis of calf vein thrombosis and no longer treat any patient solely on the basis of a clinical diagnosis, because some patients with pain and tenderness in the calf do not have venous thrombosis. Once the diagnosis is made, daily leg scans will show whether the thrombosis is progressing or regressing, thus providing an objective assessment of the effectiveness of treatment.

The ultrasound flowmeter is the simplest of the screening methods but is applicable only to the major veins.

My current policy is to divide patients with deep vein thrombosis into two groups: those with pulmonary embolism and those without. If the patient has had an embolus I order an emergency phlebogram to obtain the maximum information as soon as possible so that further emboli can be prevented. ${ }^{16}$ I approach the patient with a suspected deep vein thrombosis, but no pulmonary embolus, with both the ultrasound flowmeter and a syringe of labelled fibrinogen. If the flowmeter shows the large veins to be clear I give the fibrinogen, scan the legs the following day, and then begin treatment if the diagnosis is confirmed. The legs are observed daily with the scintillation counter, and if in spite of treatment there is any extension of the thrombus I order a phlebogram. If the flowmeter shows a major vein block phlebography is carried out immediately in case the obstructing thrombus is fresh and loose and amenable to thrombectomy.

I believe that these three techniques, which any hospital can undertake, enable us to treat deep vein thrombosis in a logical and objective manner. They should also eventually produce a tremendous increase in our knowledge and understanding of the aetiology, natural history, and treatment of this common but very serious disease.

\title{
Medical Management
}

\author{
P. T. FLUTE,* M.D., M.R.C.PATH.
}

To understand the action of anticoagulants and fibrinolytic drugs the whole process of thrombosis must be viewed as the end result of the opposing action of two forces, one leading to the deposition and the other to the removal of fibrin. Disorders of blood flow and of the vein walls, platelet adhesion and aggregation, and blood coagulation contribute to the deposition of fibrin. The fibrinolytic enzymes and cells of the reticulo-endothelial system contribute to its removal. Hence, paying attention to any one of these variables may be valuable in prophylaxis.

Once formed, fibrin may dissolve completely under the -influence of the fibrinolytic enzymes or become organized with

* Reader in Haematology, King's College Hospital Medical School, Lendon S.E.5. permanent damage to the veins. The true incidence of spontaneous thrombolysis is not yet known, but it has been found in a surprisingly high proportion of patients early in the postoperative period. ${ }^{17}$

Anticoagulants restrict the further deposition of fibrin but have no direct action on an established thrombus. Nevertheless, they may so limit its extension that they improve the chance of spontaneous thrombolysis. In assessing this objective methods must be used to measure thrombi, for recovery from clinical signs and symptoms often follows the opening of new collaterals while the thrombus remains intact.

The fibrinolytic drugs have a direct thrombolytic effect, which is achieved either by activating the fibrinolytic enzyme system or by an independent proteolysis of fibrin. 


\section{Anticoagulants}

'The orally administered coumarins and indanediones act, in competition with vitamin $\mathrm{K}$, by reducing the synthesis in the liver of prothrombin, factor VII, factor IX, and factor X. This action may be potentiated by any reduction in vitamin $\mathrm{K}$ absorption, by drugs which displace the anticoagulants from their binding sites on serum albumin, or by withdrawing drugs which increase the metabolism of the coumarins. Thus liquid paraffin reduces the absorption of the fat-soluble vitamin $\mathrm{K}$, while oral antibiotics reduce synthesis of the vitamin by the intestinal bacteria. Salicylates, phenylbutazone, chlorpromazine, clofibrate, and many other substances all compete for the binding sites on serum albumin-and thus increase the amount of free anticoagulant available. Many sedatives and tranquillizers (including the barbiturates, meprobomate, and glutethimide) increase the rate of their own metabolism and that of the coumarins by a process of "enzyme induction." When these drugs are given the anticoagulant dose has to be increased; equally, when they are withdrawn the dose must be decreased if bleeding is to be avoided as coumarin metabolism returns to normal. ${ }^{18}$ In view of the large number of drugs which can affect anticoagulant requirements frequent laboratory tests should be done when the patient's medication is changed in any way.

\section{Heparin}

Heparin directly inhibits many enzyme systems, including many coagulation enzymes, by virtue of its high content of esterified sulphuric acid and consequent strong negative charge. It is useful while the delayed effect of oral anticoagulants is awaited, and may be effective in limiting the extension of the thrombi which sometimes form even when the action of these latter drugs has developed. Continuous intravenous infusions of heparin give a more stable effect, but cause bleeding more frequently than comparable intermittent doses. ${ }^{19}$

\section{Arvin}

Arvin, an enzyme extracted from the venom of the Malayan pit viper, offers an entirely new approach to anticoagulation. ${ }^{20}$ Arvin, when injected by the intravenous or intramuscular route, converts plasma fibrinogen to microclots, which are rapidly eliminated from the circulation. Regular maintenance doses keep the plasma fibrinogen at a very low concentration and thus limit the extension of thrombi by depriving the coagulation system of its final substrate.

Arvin has no direct effect on fibrin or on the coagulation enzymes, and its administration to patients does not increase the concentration of fibrinolytic activator in their plasma. ${ }^{21-23}$ The margin of safety is high, though the first dose should be given slowly to ensure that the sudden formation of microclots does not obstruct small vessels. Bleeding is uncommon, though postoperative haemorrhage may occur if the drug is given at the time of operation or shortly after. The effects of the drug can be rapidly reversed by giving an antiserum prepared in animals and replacing the fibrinogen by transfusion. On longterm treatment a few patients have acquired resistance, probably owing to the production of antibodies to the foreign protein. ${ }^{24}$

Though anticoagulants undoubtedly reduce the incidence of fatal pulmonary embolism when given to patients with deepvein thrombosis, this protection is by no means complete. ${ }^{16}$ Moreover, there is always the risk of drug-induced haemorrhage. The selection of patients for treatment thus poses a problem, first in diagnosing the presence of a thrombus and then in a knowledge of which types are relatively safe from complications, which give rise to pulmonary embolism, and which damage the valves and later give signs of the postphlebitic syndrome or predispose to recurrent thrombosis. When thrombi of recent origin are present in the popliteal or more proximal deep veins the risk of pulnonary embolism is so great that something more than anticoagulant treatment is required. ${ }^{17}$ For such thrombi the fibrinolytic drugs offer a possible alternative to surgery.

\section{Fibrinolytic Drugs}

The most widely available fibrinolytic agent at present is $\stackrel{\vec{\rho}}{\stackrel{\vec{\rho}}{9}}$ streptokinase, purified from a mixture of streptococcal exotoxins. Because of previous streptococcal infections most individuals 음 have some antistreptokinase in their sera, but, once this antibody $\frac{\bar{\omega}}{\overrightarrow{7}}$ has been neutralized by the initial dose of streptokinase, further $\stackrel{\mathbb{Q}}{\Omega}$ streptokinase can exert its specific biochemical effect. This is to convert plasminogen, an inactive plasma globulin, to plasmin, क a proteolytic enzyme. Plasmin which is formed within a $\overrightarrow{.}$ thrombus attacks fibrin, splitting it into soluble fibrin degradation products (F.D.P.). Plasmin formed from circulating $\omega^{\circ}$ plasminogen, outside the thrombus, may also attack fibrinogen, from which it can form degradation products similar to those produced from fibrin, and many other proteins. Fortunately plasma contains an excess of antiplasmins, which normally inhibit this action of plasmin, and consequently render it specific for fibrin under physiological conditions. ${ }^{25}$

When streptokinase is given physiological concentrations of activator are often greatly exceeded and some digestion of plasma fibrinogen then occurs. The combination of the lack $\vec{\omega}$ of fibrinogen together with the anticoagulant and antiplatelet properties of the F.D.P. formed ${ }^{26}$ may cause the bleeding which is the main danger of this type of therapy. This may be avoided in one of three main ways. Firstly, by giving small doses of streptokinase directly into the thrombus. Secondly, by giving a systemic dose which is monitored by frequent laboratory tests. ${ }^{27}$ Thirdly, by giving very large systemic doses. ${ }^{28}$ In most people 500,000 units of streptokinase given in 30 minutes will neutralize their circulating antibody and also provide the excess necessary to convert rapidly all the circulating plasminogen to plasmin. If the streptokinase infusion is then continued, giving 100,000 units or more every hour, the only plasminogen available is that which can be freshly synthesized, and this is usually insufficient to allow any but a minor digestion of plasma proteins.

Increasing experience suggests that daily laboratory control (such as the easily performed thrombin clotting time) is needed to give timely warning of approaching danger. With these precautions bleeding is uncommon unless the blood vessels have been damaged. Streptokinase should not be given to patients within 72 hours of surgical operations or even longer if there are extensive areas of granulation tissue, or to those with gastrointestinal ulceration, or who have severe hypertension. Venepunctures bleed easily and normal menstrual losses are increased, sometimes dramatically.

Injection of streptokinase directly into the thrombus offers the best chance of successful thrombolysis. Nevertheless, systemic administration of large doses has usually been adopted because of the widespread nature of the thrombotic process. Rapid lysis may be obtained with some thrombi, even those obstructing major vessels, but administration may have to be continued for six days and even then is not uniformly effective. ${ }^{23}$ The best results are obtained when thrombi have been present for less than 36 hours before treatment and the worst when a recognizable stimulus to thrombosis, particularly local inflammation of the vein wall, persists. When rapid clearance of the main vessels has been obtained, follow-up shows that the function of the venous valves has often been preserved. ${ }^{2930}$ This may be of considerable importance in the prevention of local sequelae of thrombosis once the hazard to life from pulmonary embolism has passed.

After the infusion plasminogen returns to normal concentration in the plasma over 48 to 72 hours, and during this time further thrombolysis may occur. ${ }^{31}$ To reduce the risk of 
rethrombosis treatment is usually continued with oral anticoagulants. Streptokinase is antigenic and the antibody level rises appreciably in the days following an infusion. Allergic reactions are rather frequent, but can usually be controlled with corticosteroid or antihistamine drugs.

\section{Urokinase}

The allergic complications of streptokinase therapy do not occur with urokinase, a direct activator of plasminogen which can be extracted from human urine. Urokinase is not antigenic in man, and the absence of circulating antibody means that the effects of the first dose can safely be predicted according to body weight. It has a proved thrombolytic potential and has been investigated in the treatment of pulmonary embolism. ${ }^{32}$ Nevertheless, large quantities of human urine have to be used to obtain adequate supplies and hence no trial of systemic urokinase in venous thrombosis has been carried out-though local treatment may be of value. ${ }^{33}$

The precise place of fibrinolytic therapy in relation to anticoagulants and surgical procedures has yet to be defined. The most important consideration at present is to ensure that its use is limited to those with thrombi demonstrated and followed by objective methods, so that valid information is obtained.

\section{Prophylaxis}

In patients with a very high risk of pulmonary embolism, particularly the elderly in bed following trauma or tissue necrosis, prophylactic oral anticoagulants are effective in reducing mortality from thromboembolism. ${ }^{34} 35$ After general surgery the effects of encouraging mobility of the legs, raising the foot of the bed, and providing firm external support, before, during, and after operation have been investigated using the ${ }^{125}$ I-labelled fibrinogen method. The incidence of thrombosis was reduced in those over 60 years, but postoperative thrombi were still found in at least $24 \%$ of those examined. ${ }^{36}$

An antithrombotic effect has been associated with the intravenous administration of dextrans, particularly those with an average molecular weight of 70,000 , which reduce plasma viscosity and change platelet behaviour. ${ }^{37-39}$ There have been many encouraging clinical reports, while objective studies ${ }^{4041}$ have tended to confirm their effectiveness. Dextrans are contraindicated in any condition in which further expansion of plasma volume may overload the circulation, and this includes many patients in the medical wards.

In urgent need of further investigation are the drugs which alter platelet behaviour ${ }^{424}$ and those which increase the spontaneous activator of fibrinolysis in the plasma. ${ }^{44}$

\title{
Surgical Management
}

\author{
G. E. MAVOR* M.D., CH.M., F.R.C.S.ED.
}

It is useful to classify deep vein thrombosis into either peripheral or iliofemoral. The termination of the profunda femoris vein (about $5 \mathrm{~cm}$. below the groin) is the most convenient site to take as the boundary between these segments. $^{45}$ Nevertheless, the early stages of clot formation are not necessarily restricted to one or other of these segments, since both of them are often involved simultaneously. ${ }^{4-49}$ How often peripheral deep vein thrombosis extends proximally into the high segment or gives rise to pulmonary emboli is uncertain, though probably such complications are rare. On the other hand, iliofemoral venous thrombosis-though less commonis more insidious, more dangerous, and more damaging in its consequences. Moreover, this type of thrombosis is usually complicated by propagation distally. Pulmonary embolism, of ten major or fatal, occurs in about half of all cases of high segment thrombosis, while if venous occlusion occurs serious venous insufficiency is usually inevitable. ${ }^{49}$

\section{Peripheral Segment Thrombosis}

Too much significance is still placed on the peripheral segment-and particularly on the calf veins-as an initial site of thrombosis. Despite the traditional belief that intravenous clotting spreads continuously from the lower segment to the upper one, recent studies do not confirm that this is common. Thus in a combined total of 420 special necropsy studies N. M. Gibbs ${ }^{47}$ and S. Sevitt ${ }^{50}$ were unable to find a single instance of this type of spread. Likewise, in a personal series of 257 cases of iliofemoral venous thrombosis, propagation had occurred from peripheral to iliofemoral segment in only 24 instances. ${ }^{49}$ Furthermore, before the peripheral segment can be incriminated as a source of embolism, phlebography must

* Consultant Surgeon, Aberdeen Royal Infirmary, Aberdeen. be used to prove that the iliofemoral segments are clear of nonocclusive clot. The presence of acute signs of peripheral deep vein thrombosis, or direct evidence of clot formation from the results of venography, or of the ${ }^{125}$ I-tagged fibrinogen technique $^{11}$ does not remove the need to investigate the iliofemoral segments.

Past failures to prevent pulmonary embolism by ligating the superficial femoral vein below the profunda termination have been due to clot formation in the iliofemoral segment. Nevertheless, if it is confirmed that the peripheral segment is the only site of clot formation, this operation is a satisfactory procedure for controlling embolism. ${ }^{51}$ At operation noninvolvement of the iliofemoral segments should be confirmed radiologically. Ligation of the deep vein below the profunda termination is not complicated by swelling of the leg, as there are adequate " run-around" collateral channels. Nevertheless, destruction of the valves may occur and lead to one type of postphlebitic damage. ${ }^{45}$

\section{Iliofemoral Venous Thrombosis}

In iliofemoral venous thrombosis thrombus formation begins in this segment. There may be extensive formation without occlusion of the veins, and hence there is a severe risk of pulmonary embolism. ${ }^{352}$ When occlusion does occur acute venous insufficiency develops, as the venous collateral vessels are inadequate to deal with the venous return. Iliofemoral venous thrombosis with occlusion represents the most serious therapeutic challenge in deep-vein thrombosis. Since even with present day anticoagulant regimens the mortality rate of pulmonary embolism is $30 \%,{ }^{53} 54$ more attention should be paid to clearing this segment, and venous thrombectomy without ligation or plication should always be considered. 